\title{
MAGIC DISCOVERY OF VERY HIGH ENERGY EMISSION FROM THE FSRQ PKS 1222+21
}

J. Aleksić ${ }^{1}$, L. A. Antonelli ${ }^{2}$, P. Antoranz ${ }^{3}$, M. Backes ${ }^{4}$, J. A. Barrio ${ }^{5}$, D. Bastieri ${ }^{6}$, J. Becerra GonzÁlez $^{7,8}$, W. BednareK ${ }^{9}$, A. Berdyugin ${ }^{10}$, K. Berger ${ }^{7,8}$, E. Bernardini ${ }^{11}$, A. Biland ${ }^{12}$, O. Blanch ${ }^{1}$, R. K. Bock $^{13}$, A. Boller $^{12}$, G. Bonnoli ${ }^{2}$, D. Borla Tridon ${ }^{13}$, I. Braun ${ }^{12}$, T. Bretz ${ }^{14,28}$, A. Cañellas ${ }^{15}$, E. Carmona ${ }^{13}$, A. CARosi $^{2}$, P. Colin $^{13}$, E. Colombo ${ }^{7}$, J. L. Contreras ${ }^{5}$, J. Cortina ${ }^{1}$, L. Cossio ${ }^{16}$, S. Covino ${ }^{2}$, F. Dazzi ${ }^{16,29}$, A. De Angelis ${ }^{16}$, E. De Cea Del Pozo ${ }^{17}$,

B. De Lotto ${ }^{16}$, C. Delgado Mendez ${ }^{7,30}$, A. Diago Ortega ${ }^{7,8}$, M. Doert $^{4}$, A. Domínguez ${ }^{18}$, D. Dominis Prester ${ }^{19}$,

D. Dorner ${ }^{12}$, M. Doro ${ }^{20}$, D. Elsaesser ${ }^{14}$, D. Ferenc ${ }^{19}$, M. V. FonseCA ${ }^{5}$, L. Font ${ }^{20}$, C. Fruck ${ }^{13}$, R. J. GArcía LóPez ${ }^{7,8}$, M. GarczarczyK ${ }^{7}$, D. Garrido ${ }^{20}$, G. Giavitto ${ }^{1}$, N. Godinović ${ }^{19}$, D. Hadasch ${ }^{17}$, D. HäFner ${ }^{13}$, A. Herrero ${ }^{7,8}$, D. Hildebrand ${ }^{12}$, D. Höhne-Mönch ${ }^{14}$, J. Hose ${ }^{13}$, D. Hrupec ${ }^{19}$, B. Huber ${ }^{12}$, T. Jogler ${ }^{13}$, S. KLePSeR ${ }^{1}$, T. Krähenbühl ${ }^{12}$, J. Krause ${ }^{13}$, A. La Barbera ${ }^{2}$, D. Lelas ${ }^{19}$, E. LeOnARDo ${ }^{3}$, E. LindFors ${ }^{10}$, S. Lombardi ${ }^{6}$, M. LópeZ $^{5}$, E. LoRENZ $^{12,13}$, M. Makariev ${ }^{21}$, G. Maneva ${ }^{21}$, N. Mankuzhiy ${ }^{16}$, K. Mannheim ${ }^{14}$, L. Maraschi ${ }^{2}$, M. Mariotti ${ }^{6}$, M. Martínez ${ }^{1}$, D. Mazin ${ }^{1,13}$, M. Meucci ${ }^{3}$, J. M. Miranda ${ }^{3}$, R. Mirzoyan ${ }^{13}$, H. Miyamoto ${ }^{13}$, J. Moldón ${ }^{15}$, A. Moralejo ${ }^{1}$, D. Nieto ${ }^{5}$, K. Nilsson ${ }^{10,31}$, R. Orito ${ }^{13}$, I. Oya ${ }^{5}$, D. PAneque ${ }^{13}$, R. Paoletti ${ }^{3}$, S. Pardo ${ }^{5}$, J. M. Paredes ${ }^{15}$, S. Partini $^{3}$, M. Pasanen ${ }^{10}$, F. Pauss ${ }^{12}$, M. A. Perez-Torres ${ }^{1}$, M. Persic ${ }^{16,22}$, L. Peruzzo ${ }^{6}$, M. Pilia $^{23}$, J. Pochon $^{7}$, F. Prada $^{18}$, P. G. Prada Moroni $^{24}$, E. Prandini ${ }^{6}$, I. PuljaK ${ }^{19}$, I. Reichardt ${ }^{1}$, R. Reinthal ${ }^{10}$, W. Rhode ${ }^{4}$, M. Ribó ${ }^{15}$, J. Rico ${ }^{25,1}$, S. RÜGAMER $^{14}$, A. SAGgion $^{6}$, K. Saito ${ }^{13}$, T. Y. Saito ${ }^{13}$, M. Salvati ${ }^{2}$, K. Satalecka ${ }^{11}$, V. Scalzotto ${ }^{6}$, V. Scapin ${ }^{5}$, C. Schultz ${ }^{6}$ T. Schweizer $^{13}$, M. Shayduk ${ }^{13}$, S. N. Shore ${ }^{24}$, A. Sillanpä̈ ${ }^{10}$, J. Sitarek ${ }^{9}$, D. SobCZynska ${ }^{9}$, F. Spanier ${ }^{14}$, S. Spiro $^{2}$, A. Stamerra $^{3}$, B. Steinke ${ }^{13}$, J. Storz ${ }^{14}$, N. Strah ${ }^{4}$, T. Surić ${ }^{19}$, L. Takalo ${ }^{10}$, F. Tavecchio ${ }^{2}$, P. Temnikov ${ }^{21}$, T. Terzić $^{19}$, D. Tescaro $^{24}$, M. Teshima ${ }^{13}$, M. Thom ${ }^{4}$, O. Tibolla ${ }^{14}$, D. F. Torres ${ }^{25,17}$, A. Treves ${ }^{23}$, H. Vankov ${ }^{21}$, P. VogleR $^{12}$, R. M. Wagner $^{13}$, Q. WeItZEL ${ }^{12}$, V. ZABALZA ${ }^{15}$, F. ZANDANEL $^{18}$, R. ZANIN $^{1}$ (MAGIC COLlaboration)

AND

Y. T. TANAKA ${ }^{26}$, D. L. WOOD ${ }^{27}$, AND S. BUSON ${ }^{6}$

${ }^{1}$ IFAE, Edifici Cn., Campus UAB, E-08193 Bellaterra, Spain; mazin@ifae.es

2 INAF National Institute for Astrophysics, I-00136 Rome, Italy

${ }^{3}$ Dipartimento di Fisica, Università di Siena, and INFN Pisa, I-53100 Siena, Italy; antonio.stamerra@ pi.infn.it

${ }^{4}$ Fakultät für Physik, Technische Universität Dortmund, D-44221 Dortmund, Germany

${ }^{5}$ Grupo de Fisica Atlas Energias, Universidad Complutense, E-28040 Madrid, Spain

${ }^{6}$ Dipartimento di Fisica, Università di Padova and INFN, I-35131 Padova, Italy

${ }^{7}$ Inst. de Astrofísica de Canarias, E-38200 La Laguna, Tenerife, Spain; jbecerra@iac.es

${ }^{8}$ Depto. de Astrofísica, Universidad de La Laguna, E-38206 La Laguna, Spain

${ }^{9}$ Division of Experimental Physics, University of Łódź, PL-90236 Lodz, Poland

${ }^{10}$ Tuorla Observatory, University of Turku, FI-21500 Piikkiö, Finland

${ }^{11}$ Deutsches Elektronen-Synchrotron (DESY), D-15738 Zeuthen, Germany

${ }^{12}$ ETH Zurich, CH-8093, Switzerland

${ }^{13}$ Max-Planck-Institut für Physik, D-80805 München, Germany; ksaito@mpp.mpg.de

${ }^{14}$ Fakultät für Physik und Astronomie, Universität Würzburg, D-97074 Würzburg, Germany

${ }^{15}$ Facultat de Fisica, Universitat de Barcelona (ICC/IEEC), E-08028 Barcelona, Spain

${ }^{16}$ Dipartimento di Fisica Sperimentale, Università di Udine, and INFN Trieste, I-33100 Udine, Italy

${ }^{17}$ Institut de Ciències de l'Espai (IEEC-CSIC), E-08193 Bellaterra, Spain

${ }^{18}$ Inst. de Astrofísica de Andalucía (CSIC), E-18080 Granada, Spain

${ }^{19}$ Croatian MAGIC Consortium, Institute R. Boskovic, University of Rijeka and University of Split, HR-10000 Zagreb, Croatia

${ }^{20}$ Facultat de Fisica, Universitat Autònoma de Barcelona, E-08193 Bellaterra, Spain

${ }^{21}$ Inst. for Nucl. Research and Nucl. Energy, BG-1784 Sofia, Bulgaria

${ }^{22}$ INAF/Osservatorio Astronomico and INFN, I-34143 Trieste, Italy

${ }^{23}$ Dipartimento di Fisica e Matematica, Università dell'Insubria, Como, I-22100 Como, Italy

${ }^{24}$ Dipartimento di Fisica, Università di Pisa, and INFN Pisa, I-56126 Pisa, Italy

${ }^{25}$ ICREA, E-08010 Barcelona, Spain

${ }^{26}$ Institute of Space and Astronautical Science, JAXA, 3-1-1 Yoshinodai, Chuo-ku, Sagamihara, Kanagawa 252-5210, Japan

${ }^{27}$ Space Science Division, Naval Research Laboratory, Washington, DC 20375, USA

Received 2011 January 22; accepted 2011 February 11; published 2011 February 25

\section{ABSTRACT}

Very high energy (VHE) $\gamma$-ray emission from the flat spectrum radio quasar (FSRQ) PKS $1222+21$ (4C 21.35, $z=0.432)$ was detected with the MAGIC Cherenkov telescopes during a short observation $(\sim 0.5 \mathrm{hr})$ performed on 2010 June 17. The MAGIC detection coincides with high-energy MeV/GeV $\gamma$-ray activity measured by the Large Area Telescope (LAT) on board the Fermi satellite. The VHE spectrum measured by MAGIC extends from about $70 \mathrm{GeV}$ up to at least $400 \mathrm{GeV}$ and can be well described by a power-law $d N / d E \propto E^{-\Gamma}$ with a photon index $\Gamma=3.75 \pm 0.27_{\text {stat }} \pm 0.2_{\text {syst }}$. The averaged integral flux above $100 \mathrm{GeV}$ is $(4.6 \pm 0.5) \times 10^{-10} \mathrm{~cm}^{-2} \mathrm{~s}^{-1}$ $(\sim 1$ Crab Nebula flux). The VHE flux measured by MAGIC varies significantly within the 30 minute exposure implying a flux doubling time of about 10 minutes. The VHE and MeV/GeV spectra, corrected for the absorption by the extragalactic background light (EBL), can be described by a single power law with photon index $2.72 \pm 0.34$ between $3 \mathrm{GeV}$ and $400 \mathrm{GeV}$, and is consistent with emission belonging to a single component in the jet. The 
absence of a spectral cutoff constrains the $\gamma$-ray emission region to lie outside the broad-line region, which would otherwise absorb the VHE $\gamma$-rays. Together with the detected fast variability, this challenges present emission models from jets in FSRQs. Moreover, the combined Fermi/LAT and MAGIC spectral data yield constraints on the density of the EBL in the UV-optical to near-infrared range that are compatible with recent models.

Key words: cosmic background radiation - galaxies: active - galaxies: jets - gamma rays: galaxies - quasars: individual (PKS 1222+21)

\section{INTRODUCTION}

High-luminosity active galactic nuclei (AGNs) hosting powerful relativistic jets are characterized by strong nonthermal emission extending across the entire electromagnetic spectrum, from radio up to $\gamma$-rays. More than 40 AGNs have been detected in the very high energy (VHE) domain $(E>100 \mathrm{GeV})$ by ground-based Cherenkov telescopes. ${ }^{32}$ The great majority of them are BL Lac objects, while only two are classified as flat spectrum radio quasars (FSRQs): PKS 1510-08 $(z=0.36$; Wagner \& Behera 2010) and 3C $279(z=0.536$; Albert et al. 2008; Aleksic et al. 2011b), the most distant VHE source detected up to now.

FSRQs display luminous, broad emission lines often accompanied by a "big blue bump" in the optical-UV region, associated with the direct emission from the accretion disk. VHE emission from FSRQs may therefore be affected by internal absorption from the dense UV-optical radiation reprocessed in the broad-line region (BLR; Donea \& Protheroe 2003). Distant VHE quasars offer the possibility to probe the extragalactic background light (EBL), the integrated stellar and dust emission through cosmic history, in the range 0.1-10 $\mu \mathrm{m}$ (Hauser \& Dwek 2001).

The MAGIC detection (Mariotti et al. 2010) of the FSRQ PKS 1222+21 (4C 21.35, $z=0.432$; Osterbrock \& Pogge 1987) makes it the second most distant object with known redshift (after 3C 279) detected at VHE. ${ }^{33}$ PKS $1222+21$ is a $\gamma$-ray blazar (Abdo et al. 2010b) with a relatively hard spectrum in the $\mathrm{GeV}$ range and has been included in the list of $>100 \mathrm{GeV}$ emitters in the analysis of Neronov et al. (2010). It is characterized by highly superluminal jet knots with apparent velocity up to $21 \mathrm{c}$ (Lister et al. 2009).

Upper limits on the VHE emission of PKS 1222+21 have been previously derived by Whipple (Kerrick et al. 1995) at the level of $12 \times 10^{-12} \mathrm{~cm}^{-2} \mathrm{~s}^{-1}$ at $E>300 \mathrm{GeV}$. We report here on the MAGIC discovery of this source, during a phase of high activity in $\gamma$-rays announced by the Fermi/LAT Collaboration. We discuss its implications for the EBL studies and the blazar physics.

\section{OBSERVATIONS}

MAGIC consists of two $17 \mathrm{~m}$ diameter Imaging Atmospheric Cherenkov Telescopes located at the Roque de los Muchachos, Canary Island of La Palma $\left(28^{\circ} 46^{\prime} \mathrm{N}, 17^{\circ} 53^{\prime} \mathrm{W}\right)$, at the height

\footnotetext{
${ }^{28}$ Now at Ecole polytechnique fédérale de Lausanne (EPFL), Lausanne, Switzerland

${ }^{29}$ Supported by INFN Padova.

${ }^{30}$ Now at Centro de Investigaciones Energéticas, Medioambientales y

Tecnológicas (CIEMAT), Madrid, Spain.

${ }^{31}$ Now at Finnish Centre for Astronomy with ESO (FINCA), Turku, Finland.

32 For an updated list refer to http://tevcat.uchicago.edu/ or

http://www.mppmu.mpg.de/ rwagner/sources/

33 The redshift measurement $(z=0.444)$ of the VHE BL Lac 3C 66A has large uncertainties (Bramel et al. 2005).
}

of $2200 \mathrm{~m}$ a.s.l. The stereo observations provide a sensitivity ${ }^{34}$ of $0.8 \%$ of the Crab Nebula flux at $E>250 \mathrm{GeV}$ (Colin et al. 2009).

PKS 1222+21 was observed by MAGIC from 2010 May 3 to June 19 (MJD 55319 to MJD 55366) for a total of $\sim 14.3 \mathrm{hr}$. The observations started as a part of a Target of Opportunity program triggered by an increase of the flux in the Fermi passband (Donato 2010). In this Letter, we report the results obtained from the observation of June 17 (MJD 55364), when the source was detected by MAGIC in close coincidence with the brightest flare observed by the Fermi Large Area Telescope (LAT; Tanaka et al. 2011). Results from the multi-wavelength campaign covering all 2010 observations will be published elsewhere. Nevertheless, a preliminary analysis does not provide any high-significant detection with MAGIC in any other day during the campaign.

On June 17, 21:50 UT, PKS 1222+21 was observed with the MAGIC telescopes for $\sim 0.5 \mathrm{hr}$ (MJD 55364.908 to MJD 55364.931), in the so-called wobble mode. The data were taken at zenith angles between $26^{\circ}$ and $35^{\circ}$. The light conditions during the observations correspond to moderate moon light leading to a higher noise level in the data. A cleaning level higher than the standard one was therefore applied to remove signals from night sky background noise. Stereoscopic events, triggered by both MAGIC telescopes, were analyzed in the MARS analysis framework (Moralejo et al. 2009). Details on the analysis can be found in Aleksic et al. (2011a) whereas the performance of the MAGIC telescope stereo system will be discussed in detail in a forthcoming paper.

\section{RESULTS}

The strength of the signal was evaluated applying standard cuts to the PKS $1222+21$ data sample, corresponding to an energy threshold of $\approx 70 \mathrm{GeV}$ as determined by Monte Carlo events, assuming a soft spectrum with a photon index of $\Gamma=3.5$. The $\theta^{2}$ distribution (squared angular distance between the true and reconstructed source position) of the signal coming from the region of PKS $1222+21$ yields an excess of $190 \gamma$-like events (6 $\gamma$ minute $^{-1}$ ), corresponding to a statistical significance of $10.2 \sigma$ using Equation (17) in Li \& Ma (1983).

\subsection{VHE Spectrum}

The differential energy spectrum of PKS 1222+21 was reconstructed using the "Tikhonov" unfolding algorithm (Albert et al. 2007), to take into account the finite energy resolution of the instrument and the biases in the energy reconstruction. The energy spectrum, shown in Figure 1, extends up to at least $400 \mathrm{GeV}$ and is well described by a simple power law of the form

$$
\frac{d N}{d E}=N_{200}\left(\frac{E}{200 \mathrm{GeV}}\right)^{-\Gamma}
$$

\footnotetext{
${ }^{34}$ Sensitivity is defined here as the minimal integral flux to reach $5 \sigma$ signal in $50 \mathrm{hr}$ of observations.
} 


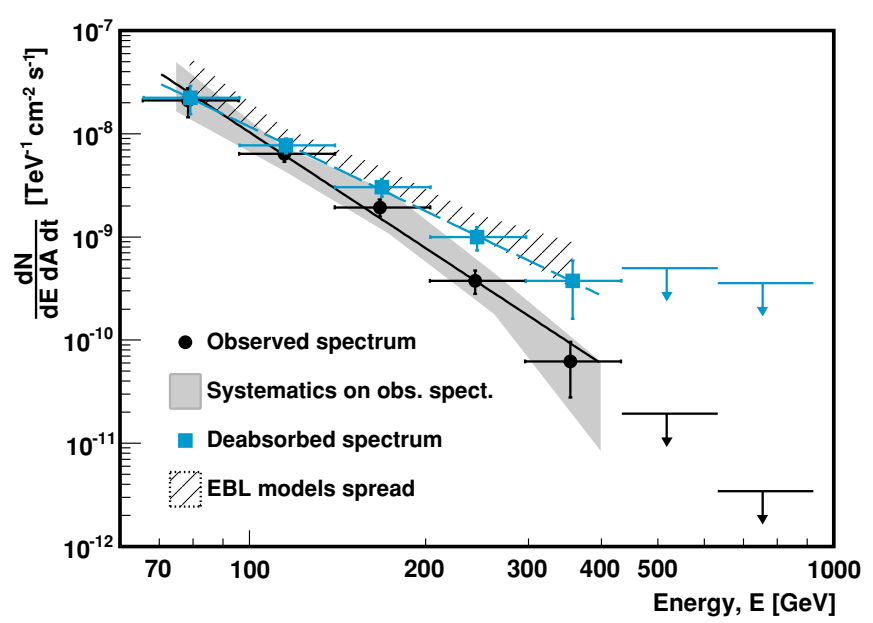

Figure 1. Differential energy spectrum of PKS $1222+21$ as measured by MAGIC on 2010 June 17. Differential fluxes are shown as black points, upper limits (95\% CL) as black arrows. The black line is the best fit to a power law. The gray shaded area represents the systematic uncertainties of the analysis. The absorption corrected spectrum and upper limits using the EBL model by Dominguez et al. (2011) are shown by the blue squares and arrows; the dashed blue line is the best-fit power law. The blue-striped area illustrates the uncertainties due to differences in the EBL models cited in the text, by Kneiske \& Dole (2010), Gilmore et al. (2009), Franceschini et al. (2008), and Albert et al. (2008).

with a photon index $\Gamma=3.75 \pm 0.27_{\text {stat }} \pm 0.2_{\text {syst }}$ and a normalization constant at $200 \mathrm{GeV}$ of $N_{200}=\left(7.8 \pm 1.2_{\text {stat }} \pm\right.$ $\left.3.5_{\text {syst }}\right) \times 10^{-10} \mathrm{~cm}^{-2} \mathrm{~s}^{-1} \mathrm{TeV}^{-1}$, yielding an integral flux $(4.6 \pm 0.5) \times 10^{-10} \mathrm{~cm}^{-2} \mathrm{~s}^{-1}(\approx 1$ Crab Nebula flux $)$ at $E>100 \mathrm{GeV}$ and $(9.0 \pm 3.6) \times 10^{-12} \mathrm{~cm}^{-2} \mathrm{~s}^{-1}(7 \%$ of the Crab Nebula flux) at $E>300 \mathrm{GeV}$, at the same level of Whipple upper limit (Section 1). For energies higher than $400 \mathrm{GeV}$ no significant excess was measured. The upper limits corresponding to $95 \%$ confidence level (CL) are shown in Figure 1. The systematic uncertainty of the analysis (studied by using different cuts and different unfolding algorithms) is shown by the gray area.

We studied the effect of the VHE $\gamma$-ray absorption due to pair production with low energy photons of the EBL by using different state-of-the-art EBL models, namely, the models by Dominguez et al. (2011), Kneiske \& Dole (2010), Gilmore et al. (2009), Franceschini et al. (2008), and the "max high UV" EBL model described in Albert et al. (2008). For each of the EBL models, the optical depth corresponding to the measured VHE $\gamma$-ray energy intervals was computed and the differential fluxes were corrected accordingly to obtain the de-absorbed (or intrinsic) spectrum. The spectrum deabsorbed with the EBL model of Dominguez et al. (2011), shown by the blue squares in Figure 1, is well fitted by a power law with an intrinsic photon index of $\Gamma_{\text {intr }}=2.72 \pm 0.34$ between $70 \mathrm{GeV}$ and $400 \mathrm{GeV}$. Uncertainties caused by the differences between the EBL models are represented in Figure 1 by the blue-striped area. The corresponding spread is smaller than the systematic uncertainties of the MAGIC data analysis.

We investigated the possible presence of a high-energy (HE) cutoff in the VHE range by fitting power laws with different photon indexes and different values for the cutoff. The method adopted is the $\chi^{2}$ difference method (see, e.g., Lampton et al. 1976). With the available statistics, at the 95\% CL we cannot exclude the presence of a cutoff above $130 \mathrm{GeV}$ for a photon index 2.4 (the lowest possible value compatible with fit uncertainties and with Fermi/LAT data, see Section 3.3) or

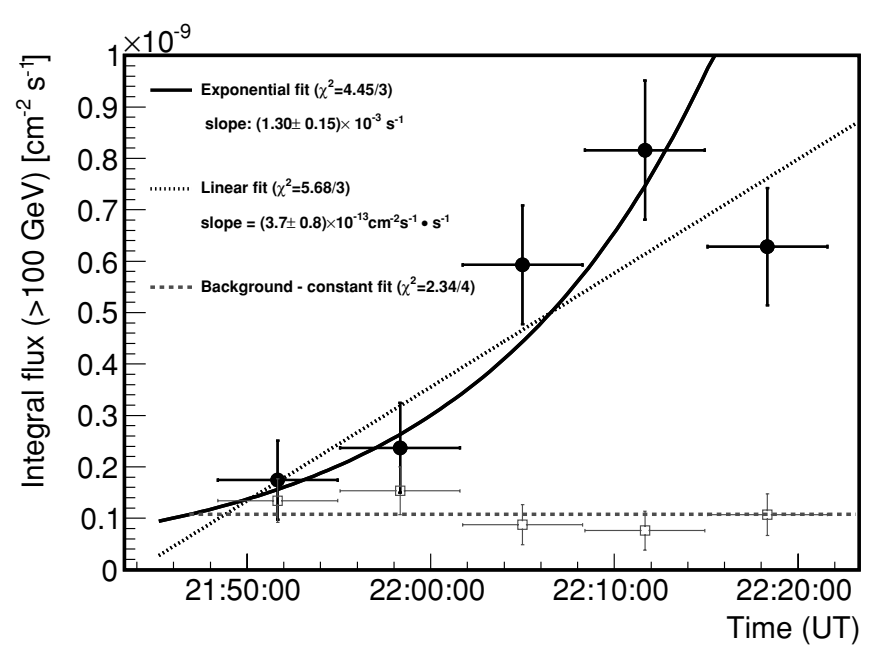

Figure 2. PKS 1222+21 light curve above $100 \mathrm{GeV}$, in 6 minutes bins (black filled circles). The observation was carried out on MJD 55364. The black solid line is a fit with an exponential function and the black dotted line a fit with a linear function. The gray open squares denote the fluxes from the background events and the gray dashed line is a fit with a constant function to these points.

above $180 \mathrm{GeV}$ for a photon index 2.7. The confidence interval is not bounded on the HE side, i.e., a fit without a cutoff is fully compatible with the data. Further observations with higher statistics are needed to better constrain the location of a possible steepening in the form of a cutoff or spectral break.

\subsection{Light Curve}

Despite the short observation time, the strength of the signal allows us to perform a variability study of the measured integral fluxes above $100 \mathrm{GeV}$. The light curve binned in 6 minutes long intervals is shown in Figure 2 and reveals clear flux variations. The constancy hypothesis $\left(\chi^{2} / \mathrm{NDF}=28.3 / 4\right)$ is rejected with high confidence (probability $<1.1 \times 10^{-5}$ ). The fluxes of background events surviving the $\gamma /$ hadron selection cuts are compatible with being constant, and hence we can exclude a variation of the instrument performance during the observation.

To quantify the variability timescale we performed an exponential fit (solid black line in Figure 2). A linear fit is also acceptable but does not allow us to define a timescale unambiguously. For the exponential fit the doubling time of the flare is estimated as $8.6_{-0.9}^{+1.1}$ minutes. The derived timescale corresponds to the fastest time variation ever observed in an FSRQ in the VHE range and in any other energy range (Foschini et al. 2011), and is among the shortest timescales measured on $\mathrm{TeV}$ emitting sources (Abramowski et al. 2010).

\subsection{The HE-VHE SED}

In the $\mathrm{HE} \mathrm{MeV/GeV} \mathrm{energy} \mathrm{range} \mathrm{measured} \mathrm{by} \mathrm{Fermi/LAT}$ the source showed a significant flare lasting $\sim 3$ days, with a flux peak on 2010 June 18 (MJD 55365) (Tanaka et al. 2011). A dedicated analysis found that the $1 / 2 \mathrm{hr}$ MAGIC observation fell within a gap in the LAT exposure, thus we analyzed a period of $2.5 \mathrm{hr}$ (MJD 55364.867 to 55364.973), encompassing the MAGIC observation. The LAT analysis for this time bin was performed as in Tanaka et al. (2011), where details can be found. It results in an integral flux $(6.5 \pm 1.9) \times 10^{-6} \mathrm{~cm}^{-2} \mathrm{~s}^{-1}$ at energy $E>100 \mathrm{MeV}$. The observation in such a short time does not provide any detection with Fermi/LAT at $E>2 \mathrm{GeV}$. Two Fermi/LAT spectral points up to $2 \mathrm{GeV}$ together with an upper limit at the $95 \% \mathrm{CL}$ in the range $2-6.3 \mathrm{GeV}$ are combined 


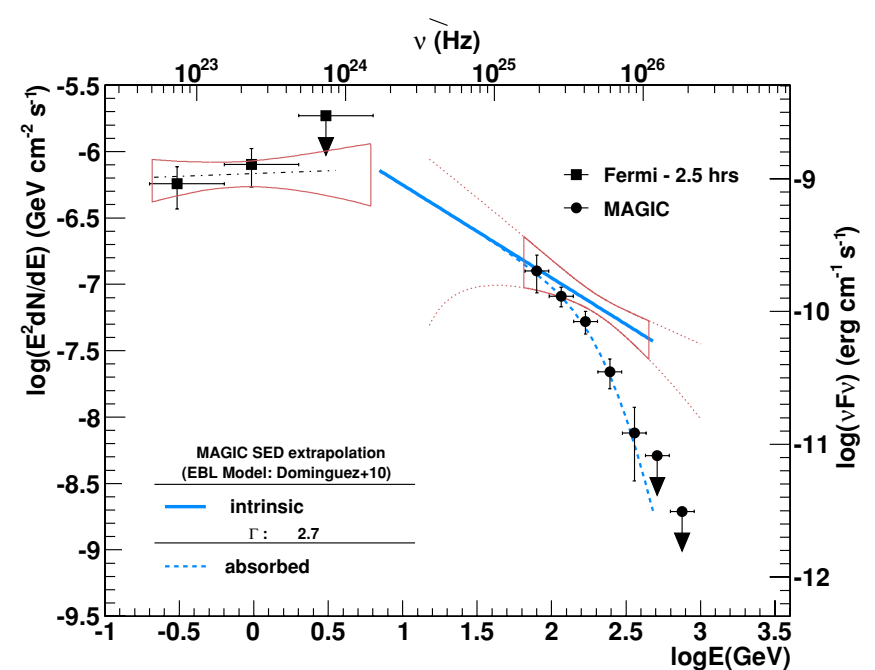

Figure 3. High-energy SED of PKS 1222+21 during the flare of 2010 June 17 (MJD 55364.9), showing Fermi/LAT (squares) and MAGIC (circles) differential fluxes. A red bow tie in the $\mathrm{MeV} / \mathrm{GeV}$ range represents the uncertainty of the likelihood fit to the Fermi/LAT data. The unfolded and deabsorbed spectral fit of the MAGIC data is also shown as a red bow tie, extrapolated to lower and higher energies (dotted lines) according to Abdo et al. (2009). A thick solid line (photon index $\Gamma=2.7$ ) indicates a possible extrapolation of the MAGIC deabsorbed data to lower energies. The thick dashed line represents the EBL absorbed spectrum obtained from the extrapolated intrinsic spectrum using the model by Dominguez et al. (2011).

with the MAGIC data in the spectral energy distribution (SED) shown in Figure 3.

The figure also shows bow ties representing uncertainties associated with the spectral fits. The Fermi/LAT spectrum is best described by a single power law with index of $1.95 \pm 0.21$. In the case of MAGIC data the bow tie refers to the "intrinsic" source spectrum, i.e., to the observed spectrum corrected for EBL absorption, described in Section 3.1. An extrapolation of the intrinsic spectrum in the MAGIC range to lower energies is also shown indicating that: (1) there is a potentially smooth connection between the Fermi/LAT and MAGIC extrapolated data in the 3 to $10 \mathrm{GeV}$ region and (2) the photon index steepens from 1.9 in the Fermi/LAT range to 2.7 in the MAGIC range. These results agree with the analysis of wider temporal intervals during this flare and during the whole active period, in which the source spectrum is well described by a broken power law with an energy break falling between 1 and $3 \mathrm{GeV}$ (Tanaka et al. 2011). Furthermore, it is found that the HE tail $(E>2 \mathrm{GeV})$ of the Fermi/LAT spectrum of PKS $1222+21$ extends up to $50 \mathrm{GeV}$, with a photon index in the range 2.4-2.8.

\section{DISCUSSION}

\subsection{EBL Limits}

The interaction of VHE $\gamma$-rays with low energy photons of the isotropic EBL is a process with an energy dependent threshold, thus leading to an imprint of the EBL density on the measured VHE $\gamma$-ray spectra of extragalactic sources (Mazin \& Raue 2007). For PKS $1222+21(z=0.432)$, the measured spectrum spans from $70 \mathrm{GeV}$ to $400 \mathrm{GeV}$ probing EBL photons in the range $0.1-1 \mu \mathrm{m}$ (i.e., UV to near-infrared).

The EBL constraints using VHE $\gamma$-rays are usually derived assuming an intrinsic spectrum of the source (e.g., Aharonian et al. 2006). In FSRQs, the presence of dense radiation fields of soft photons can lead to the internal absorption of VHE $\gamma$-rays, mimicking harder-than-intrinsic spectra (e.g., Sitarek \&

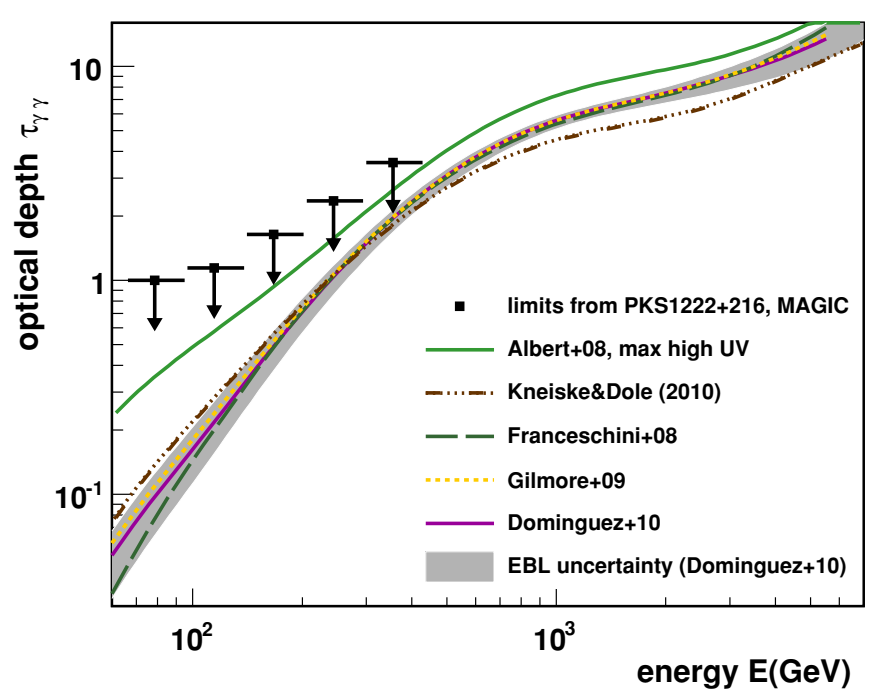

Figure 4. Optical depth along the line of sight to PKS $1222+21(z=0.432)$ for a range of EBL models and the limits $(95 \%$ CL) from the MAGIC measurement, assuming the limiting intrinsic photon index $\Gamma_{\mathrm{VHE}}=2.4$. The gray shaded area shows the uncertainties in the EBL determination as derived in Dominguez et al. (2011), Section 6.1 and Figure 13.

Bednarek 2008). However, for realistic spectral distributions of the internal photon fields it should not change the EBL limits significantly (Tavecchio \& Mazin 2009).

In our case the simultaneous data from Fermi/LAT, which is free from internal or external absorptions, have been used to constrain the intrinsic photon index in VHE (e.g., Georganopoulos et al. 2010; Finke \& Razzaque 2009). We adopt a method similar to the one utilized by Georganopoulos et al. (2010): the intrinsic spectrum in the VHE regime is assumed to follow the extrapolation of the Fermi/LAT above $3 \mathrm{GeV}$ with a $\Gamma=2.4$. This is a conservative assumption since in reality the spectrum could soften with increasing energy.

The upper limit (95\% CL) on the optical depth, $\tau_{\max }$, for VHE $\gamma$-rays can be obtained from

$$
\tau_{\max }(E)=\log \left[\frac{F_{\text {intr }}(E)}{F_{\text {obs }}(E)-1.64 \cdot \Delta F(E)}\right],
$$

where $F_{\text {intr }}(E)$ is the maximum intrinsic flux at energy $E$, and $F_{\text {obs }}(E)$ and $\Delta F(E)$ are the MAGIC measured flux and its error, respectively. The maximum intrinsic flux has been normalized at $70 \mathrm{GeV}$ assuming the EBL model giving a maximum flux absorption of $30 \%$ (Albert et al. 2008). The derived limits on the optical depth are shown in Figure 4 together with a compilation of the predicted optical depths for a source at $z=0.432$ computed according to recent EBL models. The limits confirm previous constraints on the EBL models in the UV to nearinfrared regimes derived using VHE (Aharonian et al. 2006; Mazin \& Raue 2007; Albert et al. 2008) and HE spectra (Abdo et al. 2010a). Given the fact that the EBL models predict for this redshift a stronger absorption with increasing energy, our data do not indicate a softening of the spectrum within the energy range of our observations.

\subsection{VHE $\gamma$-ray Emission}

In the framework of the currently accepted EBL models, the observed simultaneous $\mathrm{VHE}$ and $\mathrm{GeV}$ spectra are consistent with a single power law with index $\sim 2.7 \pm 0.3$ between $3 \mathrm{GeV}$ and $400 \mathrm{GeV}$, without a strong intrinsic cutoff. This evidence 
suggests that the $100 \mathrm{MeV}-400 \mathrm{GeV}$ emission belongs to a unique component, peaking at $\approx 2-3 \mathrm{GeV}$, produced in a single region of the jet. If the emission process is inverse Compton (IC) scattering on external photons by relativistic electrons in the jet, as commonly assumed, a strong softening of the spectrum is expected above few tens of $\mathrm{GeV}$ if the external photons derive from the BLR. This is due to the combination of two effects: the decreased efficiency of the IC scattering occurring in the Klein-Nishina (KN) regime (e.g., Ghisellini \& Tavecchio 2009) and the absorption of $\gamma$-rays through pair production (Reimer 2007; Tavecchio \& Mazin 2009; Liu \& Bai 2006). ${ }^{35}$

The energy above which the $\mathrm{KN}$ effects become important can be roughly expressed as $E_{\mathrm{KN}} \simeq 22.5 v_{o, 15}^{-1} \mathrm{GeV}$, where $v_{o, 15}$ is the frequency of the target photons in units of $10^{15} \mathrm{~Hz}$ (or $E_{\mathrm{KN}} \simeq 75 \lambda_{\mu \mathrm{m}} \mathrm{GeV}$ in wavelength $\mu \mathrm{m}$ units). $\gamma$-ray absorption becomes effective when $E_{\gamma \gamma} \simeq 60 \nu_{o, 15}^{-1} \mathrm{GeV}$ $\left(E_{\gamma \gamma} \simeq 200 \lambda_{\mu \mathrm{m}} \mathrm{GeV}\right)$. Above this energy a cutoff is then expected. The importance of both effects in the $10-100 \mathrm{GeV}$ band is reduced if the external photon field is associated with the IR torus $\left(v_{\mathrm{o}}=10^{13} \mathrm{~Hz}\right)$, as envisioned by the "far dissipation" scenarios (e.g., Sikora et al. 2008). In that case both effects start to be important above $\approx 1 \mathrm{TeV}$. The absence of a spectral break or cutoff in the spectrum observed by MAGIC strongly suggests that the $\gamma$-ray emission is not produced within the BLR.

The other important result of the MAGIC observation is the evidence of fast variability, $t_{\mathrm{var}} \sim 10$ minutes, indicating an extremely compact emission region with transverse dimensions, $R \sim 1.3 \times 10^{14}(\delta / 10)\left(t_{\mathrm{var}} / 10\right.$ minutes $) \mathrm{cm}$. This seems to be difficult to reconcile with the "far dissipation" scenarios if the emission takes place in the entire cross section of the jet (see also Tavecchio et al. 2010). Estimating the size of the $\mathrm{BLR}, R_{\mathrm{BLR}}$ from the accretion disk luminosity, $L_{\mathrm{disk}}=$ $5 \times 10^{45} \mathrm{erg} \mathrm{s}^{-1}$ (Fan et al. 2006), the distance of the emitting region is expected to be around $d>R_{\mathrm{BLR}}=3 \times 10^{17} \mathrm{~cm}$. Assuming a conical jet with constant opening angle $\theta_{\mathrm{j}}$ (see, however, the suggestion of recollimation; Marscher 1980), its size would be $R \sim \theta_{\mathrm{j}} d \sim 3 \times 10^{16}\left(\theta_{\mathrm{j}} / 5^{\circ}\right) \mathrm{cm}$. The absence of absorption features in the VHE spectrum allows also to exclude absorption within the emitting region and, together with the observed variability, to put a lower limit to the Doppler factor of the source. From Dondi \& Ghisellini (1995), Equation (3.7), assuming a power-law photon index 1.5 for the spectrum of the optical target photons, we get a lower limit $\delta>15$, in agreement with Doppler factors derived from radio observations (Section 1).

A possibility to reconcile the spectral information (pointing to emission beyond the BLR) and the fast variability is to invoke the presence of very compact emission regions embedded within the large-scale jet, as already proposed by several authors to explain the exceptionally rapid variability in PKS 2155-304, Mkn 501, and AO 0235+164 (Ghisellini \& Tavecchio 2008; Giannios et al. 2009; Marscher \& Jorstad 2010). An alternative possibility is that the jet experiences a strong recollimation forming a small emitting nozzle (e.g., Nalewajko \& Sikora 2009) as already suggested for M87 and PKS 2155-304 (e.g., Bromberg \& Levinson 2009; Stawarz et al. 2006). Alternative scenarios involving proton-driven cascades or proton-synchrotron emission in amplified magnetic fields, e.g., generated by filamentation instabilities (Frederiksen et al. 2010), could also play a role.

\footnotetext{
35 We note that this absorption has been invoked by Poutanen \& Stern (2010) to explain the existence of an apparently "universal" break energy in the $\gamma$-ray spectrum of FSRQs at $2 \mathrm{GeV}$.
}

In conclusion, the MAGIC observations of VHE emission from the FSRQ PKS $1222+21$ put severe constraints on emission models of blazar jets. These results were obtained from a short observation of a flaring source thanks to the collaboration between the MAGIC and Fermi projects. Repeated and hopefully longer observations of flaring blazars with MAGIC and Fermi promise substantial progress in the study of extreme blazars.

We thank the Instituto de Astrofísica de Canarias for the excellent working conditions at the Observatorio del Roque de los Muchachos in La Palma. The support of the German BMBF and MPG, the Italian INFN, the Swiss National Fund SNF, and the Spanish MICINN is gratefully acknowledged. This work was also supported by the Marie Curie program, by the CPAN CSD2007-00042 and MultiDark CSD2009-00064 projects of the Spanish Consolider-Ingenio 2010 programme, by grant DO02-353 of the Bulgarian NSF, by grant 127740 of the Academy of Finland, by the YIP of the Helmholtz Gemeinschaft, by the DFG Cluster of Excellence "Origin and Structure of the Universe," and by the Polish MNiSzW Grant N N203 390834.

The Fermi/LAT Collaboration acknowledges support from a number of agencies and institutes for both development and the operation of the LAT as well as scientific data analysis. These include NASA and DOE in the United States, CEA/Irfu and IN2P3/CNRS in France, ASI and INFN in Italy, MEXT, KEK, and JAXA in Japan, and the K. A. Wallenberg Foundation, the Swedish Research Council and the National Space Board in Sweden. Additional support from INAF in Italy and CNES in France for science analysis during the operations phase is also gratefully acknowledged.

\section{REFERENCES}

Abdo, A. A., et al. (Fermi/LAT Collaboration) 2009, ApJ, 707, 1310 Abdo, A. A., et al. (Fermi/LAT Collaboration) 2010a, ApJ, 723, 1082 Abdo, A. A., et al. (Fermi/LAT Collaboration) 2010b, ApJS, 183, 46 Abramowski, A., et al. (HESS Collaboration) 2010, A\&A, 520, 83 Aharonian, F., et al. 2006, Nature, 440, 1018

Albert, J., et al. (MAGIC Collaboration) 2007, Nucl. Instrum. Methods Phys. Res. A, 583, 494

Albert, J., et al. (MAGIC Collaboration) 2008, Science, 320, 1752

Aleksic, J., et al. (the MAGIC Collaboration) 2011a, ApJ, 726, 58

Aleksic, J., et al. (the MAGIC Collaboration) 2011b, A\&A, submitted (arXiv:1101.2522)

Bromberg, O., \& Levinson, A. 2009, ApJ, 699, 1274

Bramel, D. A., et al. 2005, ApJ, 629, 108

Colin, P., et al. (for the MAGIC Collaboration) 2009, in Proc. 31st ICRC, Lodz, Poland, July 2009 (arXiv:0907.0960)

Dominguez, A., et al. 2011, MNRAS, 410, 2556

Donato, D. (Fermi/LAT Collaboration) 2010, ATel, 2584

Dondi, L., \& Ghisellini, G. 1995, MNRAS, 273, 583

Donea, A.-C., \& Protheroe, R. J. 2003, Astropart. Phys., 18, 377

Fan, Z., Cao, X., \& Gu, M. 2006, ApJ, 646, 8

Finke, J., \& Razzaque, S. 2009, ApJ, 698, 1761

Foschini, L., Ghisellini, G., Tavecchio, F., Bonnoli, G., \& Stamerra, A. 2011, MNRAS, submitted (arXiv:1101.1085)

Franceschini, A., Rodighiero, G., \& Vaccari, M. 2008, A\&A, 487, 837

Frederiksen, J. T., Haugblle, T., Medvedev, M. V., \& Nordlund, A.. 2010, ApJ, 722, L114

Georganopoulos, M., Finke, J. D., \& Reyes, L. C. 2010, ApJ, 714, L157

Ghisellini, G., \& Tavecchio, F. 2008, MNRAS, 386, 28

Ghisellini, G., \& Tavecchio, F. 2009, MNRAS, 397, 985

Giannios, D., Uzdensky, D. A., \& Begelman, M. C. 2009, MNRAS, 395, 29

Gilmore, R. C., Madau, P., Primack, J. R., Somerville, R. S., \& Haardt, F 2009, MNRAS, 399, 1694

Hauser, M. G., \& Dwek, E. 2001, ARA\&A, 39, 249 
Kerrick, A. D., et al. (Whipple Collaboration) 1995, ApJ, 452, 588

Kneiske, T. M., \& Dole, H. 2010, A\&A, 515, 19

Lampton, M., Margon, B., \& Bowyer, S. 1976, ApJ, 208, 177

Li, T.-P., \& Ma, Y.-Q. 1983, ApJ, 272, 317

Lister, M. L., et al. 2009, AJ, 138, 1874

Liu, H. T., \& Bai, J. M. 2006, ApJ, 653, 1089

Mariotti, M., et al. (the MAGIC Collaboration) 2010, ATel, 2684

Marscher, A. P. 1980, ApJ, 235, 386

Marscher, A. P., \& Jorstad, S. G. 2010, in Proc. xFermi Meets Jansky (arXiv:1005.5551)

Mazin, D., \& Raue, M. 2007, A\&A, 471, 439

Moralejo, A., et al. (for the MAGIC Collaboration) 2009, in Proc. 31st ICRC, Lodz, Poland, July 2009 (arXiv:0907.0943)

Nalewajko, K., \& Sikora, M. 2009, MNRAS, 392, 1205
Neronov, A., Semikoz, D. V., \& Vovk, Ie. 2010, arXiv:1004.3767

Osterbrock, D. E., \& Pogge, R. W. 1987, ApJ, 323, 108

Poutanen, J., \& Stern, B. 2010, ApJ, 717, L118

Reimer, A. 2007, ApJ, 665, 1023

Sikora, M., Moderski, R., \& Madejski, G. M. 2008, ApJ, 675, 71

Sitarek, J., \& Bednarek, W. 2008, MNARS, 391, 624

Stawarz, L., Aharonian, F., Kataoka, J., Ostrowski, M., Siemiginowska, A., \& Sikora, M. 2006, MNRAS, 370, 981

Tanaka, Y. T., et al. 2011, ApJ, in press

Tavecchio, F., Ghisellini, G., Bonnoli, G., \& Ghirlanda, G. 2010, MNRAS, 405, L94

Tavecchio, F., \& Mazin, D. 2009, MNRAS, 392, L40

Wagner, S., \& Behera, B. 2010, in 10th HEAD Meeting, Hawaii (BAAS, 42, 2 , $07.05)$ 